\title{
Eigenfunctions for a Quantum Wire on a Single Electron at Its Surface and in the Quantum Well with Beaded Fractional Quantized States for the Fractional Charges
}

\author{
Saleem Iqbal' ${ }^{1}$, Farhana Sarwar ${ }^{1,2}$, Syed Mohsin Raza ${ }^{3}$ \\ ${ }^{1}$ Department of Mathematics, University of Balochistan, Quetta, Pakistan \\ ${ }^{2}$ Department of Mathematics, F. G. Girls Degree College, Quetta, Pakistan \\ ${ }^{3}$ Department of Physics, University of Balochistan, Quetta, Pakistan \\ Email: saleemiqbal81@yahoo.com,f_saleem10@yahoo.com,smraza7@yahoo.com
}

Received 10 January 2016; accepted 22 February 2016; published 25 February 2016

Copyright (C) 2016 by authors and Scientific Research Publishing Inc.

This work is licensed under the Creative Commons Attribution International License (CC BY). http://creativecommons.org/licenses/by/4.0/

\section{(c) (i) Open Access}

\section{Abstract}

We developed energy profiles for the fractional quantized states both on the surface of electron due to overwhelming centrifugal potentials and inside the electron at different locations of the quantum well due to overwhelming attractive electrodynamic potentials. The charge as a physical constant and single entity is taken as density and segments on their respective sub-quanta (floats on sub quanta) and hence the fractional charge quantiz at in. There is an integrated oscillatory effect which ties all fractional quantized states both on the surface and in the interior of the volume of an electron. The eigenfunctions, i.e., the energy profiles for the electron show the shape of a string or a quantum wire in which fractional quantized states are beaded. We followed an entirely different approach and indeed thesis to reproducing the eigenfunctions for the fractional quantized states for a single electron. We produced very fascinating mathematical formulas for all such cases by using Hermite and Laguerre polynomials, spherical based and Neumann functions and indeed asymptotic behavior of Bessel and Neumann functions. Our quantization theory is dealt in the momentum space.

\section{Keywords}

Fractional Charge Quantization, Fractional Fourier Transform, Hermite Polynomials, Sub Quanta of Electron, Spherical Bessel and Neumann Functions, Lagueree Polynomials 


\section{Introduction}

The inside of the electron is treated like a potential well with its depth equivalent to radius $r_{e}$ and that the charge above it is fractionally quantized. The bounded electron having orbit angular and rotational momentum will have a continuous change of its surface, as a consequence of which, charges as a constant entity will rearrange in its fragmented form and hence the fractional quantized states above its surface. Each fractional quantized state is a manifestation of sub quanta due to change in mass distribution. This happens in the momentum space just like the Compton and Photoelectric effects. We are refereing to momentum space because some kind of external momentum, may be due to photons, will cause the charge on an electron to become degenerate, of course, only above the surface. The fractional charge floats on the surface of sub quanta (energy becomes oscillatory due to segmented masses of electron but coupled with a string or a quanta wire). The question why and how this happens could be answered by considering that the centrifugal potential is dominating over the electrodynamic potential, as a consequence of which,quantum well with a depth $r_{e}$ is becoming shallow and that the segmented masses of electron are shifting on the surface but coupled with a string. This coupling, in fact, is due to gyroscopic behaviour of an electron. The coupling constant $\left(\frac{e^{2}}{\hbar^{\prime} c} \sim \frac{1}{137}\right)$ is overwhelmed by the gyroscopic constant, $\frac{g^{2}}{\hbar c}(0.2-0.8)$. The gyroscopic behaviour of an electron causes the constant entity of charge to become degenerate and fractionally quantized above its surface (of course due to segmented mass distribution but coupled with a string at different locations just above the surface).

The radial eigen function $R_{E l}$ inside the quantum well (with depth equivalent to radius of an electron) follows strips of whirling pool as a manifestation of competing centrifugal force and attractive electrodynamic potentials. The charges always reside either on the surface of the electron or on the surface of a quanta.With this competing behaviour the shape of the surface of an electron is changed preferably due to the shallowing of quantum well. The brim of quantum well becomes effective due to overwhelming behaviour of the centrifugal potential over the attractive electrodynamic potential and hence the spitting of the segmented masses (sub quanta) above the surface. But all the erupted segmented masses are coupled with a central force and indeed with a string. Each segmented mass of an electron is equivalent to its sub quanta. These sub quanta of electrons are tied with each other with a gyroscopic behaviour. The charge is also fragmented and floats on their respective sub quanta.

\section{Theory}

For $r<r_{e}$, i.e..,less than the radius or depth of the quantum well of an electron, the radial eigen function follows the equation [1]-[3].

$$
\begin{aligned}
& {\left[\frac{-\hbar^{2}}{2 \mu}\left(\frac{\mathrm{d}^{2}}{\mathrm{~d} r}+\frac{2}{r} \frac{\mathrm{d}}{\mathrm{d} r}\right)+\frac{\ell(\ell+1) \hbar^{2}}{2 \mu r^{2}}\right] R_{E, \ell}(r)=\left(E+V_{0}\right) R_{E, \ell}(r)} \\
& \Rightarrow\left[-\left(\frac{\mathrm{d}^{2}}{\mathrm{~d} r}+\frac{2}{r} \frac{\mathrm{d}}{\mathrm{d} r}\right)+\frac{\ell(\ell+1)}{r^{2}}\right] R_{E l}(r)=\frac{2 \mu}{\hbar^{2}}\left(E+V_{0}\right) R_{E, \ell}(r)
\end{aligned}
$$

where $\mu$ is the reduced or segmented masses, $\frac{\ell(\ell+1) \hbar^{2}}{2 \mu r^{2}}$ the centrifugal potential on each strip of the whirling pool inside the electron (quantum well), $-\frac{e^{2}}{r_{e}}=V_{0}$ the electrodynamic potential and $l$ the azimuthal quantum number (determines the shape of the whirling strips in the quantum well). We roughly estimated the radius or depth of the quantum well of an electron by considering the charge as density of electron $\left(r_{e} \sim 10-15 \mu \mathrm{m}\right)$. Our assumption for treating the charge as density is due to very small volume of the electron.

Each of the broken sub quanta are woven in a string due to whirling and swirling effects (electro weak interactions) on an electron and is manifestation of gyroscopic behaviour. Equation (1) can be rewritten as 


$$
\left[-\left(\frac{\mathrm{d}^{2}}{\mathrm{~d} r}+\frac{2}{r} \frac{\mathrm{d}}{\mathrm{d} r}\right)+\frac{\ell(\ell+1)}{r^{2}}\right] R_{E \ell}(r)=K^{2} R_{E, \ell}(r)
$$

where

$$
K=\left[\frac{2 \mu}{\hbar^{2}}\left(E+V_{0}\right)\right]^{1 / 2} \text { and } V_{0}=-\frac{e^{2}}{r_{e}}
$$

When broken sub quanta above the surface of an electron are woven in a string, they should have an integrated oscillatory effects.

Writing

$$
\psi_{n}=\frac{1}{\sqrt{2^{n}} n ! \sqrt{\pi}} \mathrm{e}^{-\frac{x^{2}}{2}} H_{n}(x)
$$

The exponent $n$ of 2 will have fractional quantization, $0.1 \leq n_{f} \leq 0.9$ where as $n !=0 !=1$ for integrated oscillatory behaviour. This would work for Equation (4). The quantum of dipole moment leads to charge quantization [4]-[7]

$$
x=h q
$$

where $x$ = quantum dipole moment, $h$ is Planck's constant(quantum action) and $q$ the charge.

The matter energy such as of an electron exists in the form of transverse wave.This is oscillatory (quantum action) and configures a space called a wave packet or "quanta". We consider that the charge on an electron is treated as its density which is not only smeared on the surface but also inside the volume despite the fact that charges always reside on the surface. This is the reason that fractional charges float on their respective segmented masses above the surface of an electron. Considering the symmetry of the harmonic oscillator potential,solution for eigen function exists in the form

$$
\psi_{\ell, m}=R(r) \psi_{\ell}^{m}(\Theta, \Phi)
$$

in which the differential equation for the radial eigen function (on the surface of an electron) $R(r)$ is

$$
\frac{1}{r^{2}} \frac{\mathrm{d}}{\mathrm{d} r}\left(r^{2} \frac{\mathrm{d} R}{\mathrm{~d} r}\right)+\frac{2 m}{\hbar^{2}}[E-V(r)]=\frac{\ell(\ell+1)}{r^{2}} R(r), r=r_{e}
$$

Modifying Equation (4) with Equation (5) for fractional charge quantized states having an integrated oscillatory effect at the surface of an electron.

$$
\psi_{n_{f}}=\frac{1}{\sqrt{2^{n_{f}} 0 ! \sqrt{\pi}}} \mathrm{e}^{-\left(\frac{h q}{\sqrt{2}}\right)^{2}} H_{n_{f}}(h q)
$$

We have calculated $H_{n_{f}}(h q)$ for $0.1 \leq n_{f} \leq 0.9$ and reproduced the shape of the eigen function on the surface of the electron [7], i.e., case I $\left(r=r_{e}\right)$.

\subsection{Case I $\left(r=r_{e}\right)$}

$$
\psi_{n_{f}}(h q)=\left(\frac{0.17 \leq \alpha \leq 1.53}{2^{n_{f}} 0 ! \sqrt{\pi}}\right)^{\frac{1}{2}} \mathrm{e}^{-\alpha^{2}\left(\frac{h q}{\sqrt{2}}\right)} H_{n_{f}}(\alpha h q)
$$

where $\alpha$ is a rotation vector to locate the fractional quantized states on the surface of an electron. The rotation vector, “ $\alpha$ ” in complex plane $(t, w)$ is determined from the Fractional Fourier Transform analysis [8]. we found $H_{n_{f}}(\alpha h q)=2^{n_{f}}$ for the fractional quantized states, $0.1 \leq n_{f} \leq 0.9$ with $0.17 \leq \alpha \leq 1.53$ [8]. The term in Equation (9), i.e..,

$$
\exp \left[-\alpha^{2}\left(\frac{h q}{\sqrt{2}}\right)^{2}\right] \equiv \exp -\alpha^{2}
$$


tends to unity and is valid for Equation (8). The reason for the convergence in the above expression is explained in [8]. The eigenfunction $\psi_{n_{f}}(h q)$ in Equation (9) with oscillatory function $H_{n_{f}}(\alpha h q)$, Hermite function, shows the energy profile as a straight line with fractional quantum states and that the profile is a tangent on the surface of an electron. The fractional quantum states $n_{f}$ are beaded in a string (quantum wire in the form of a straight line energy profile). $\mathrm{e}^{i_{f} \alpha}$, when plotted against, $n_{f}$ the fractional quantized states, shows the logarithmic profile for the rotation vector, $\alpha$ [9]. We will have the admissible solution of Equation (7) in the form

$$
r^{\ell} \equiv r_{e}^{\ell}
$$

Writing the series for Equation (10), i.e..,

$$
\phi=-\sum_{k=1}^{\infty} a_{k} r_{e}^{k}
$$

Using this series Equation (11) and making it to terminate with asymptotic condition, we have the recurrence relation (to reproduce the more appropriate shape of the string on the surface of an electron).

$$
\begin{gathered}
a_{k}=\frac{a(k-2)-(\lambda-3)}{(k-\ell)(k+\ell+1)} a_{k-2}, k>\ell+1 \\
\lambda=2 n_{f}+1 \text { asymptotic condition. }
\end{gathered}
$$

and Ultimately,we get the series in the form

$$
\begin{gathered}
\phi=r_{e}^{\ell} \times\left(\text { power series in }\left(\frac{h q}{\sqrt{2}}\right)^{2}\right) \\
\phi=r_{e}^{\ell} \times \mathrm{e}^{-r^{2}}=r_{e}^{\ell} \times \mathrm{e}^{-\left(\frac{h q}{\sqrt{2}}\right)^{2}}
\end{gathered}
$$

When centrifugal potential dominates over the attractive electrodynamic potential in the quantum well with its depth equal to radius of an electron swirling causes the strips of the whirlpool to change into elliptic orbits with enhanced Eulerian angles. Thus we have

$$
\psi_{n_{f}, \ell, m}=\mathrm{e}^{-\left(\frac{h q}{\sqrt{2}}\right)^{2}} \phi_{n_{f}}^{l}(r) Y_{l}^{m}(\Theta, \Phi) ; n_{f} \geq \ell, 0.1 \leq n_{f} \leq 0.9
$$

With Equation (14), $\phi_{n_{f}}^{\ell}(r)$ will change into Lagueree polynomials. Solution of Equation (14) becomes more complicated. We shall have a shallowing of a quantum well continuously, therefore,treatment of $Y_{\ell}^{m}(\Theta, \Phi)$ along with Eulerian angle would becomes cumbersome. This problem can be overcome by defining

$$
\rho=K r
$$

and

$$
R_{\ell}(\rho) \equiv R_{E, \ell}(r)
$$

With Equation (15) and $r<r_{e}$ (less than the depth of the quantum well),the radial eigen function $R_{e}(\rho)$ satisfies the spherical Bessel differential equation [1]-[3]

$$
\left[\frac{\mathrm{d}^{2}}{\mathrm{~d} \rho^{2}}+\frac{2}{\rho} \frac{\mathrm{d}}{\mathrm{d} \rho}+\left(1-\frac{\ell(\ell+1)}{\rho^{2}}\right)\right] R_{\ell}(\rho)=0 .
$$

The particular solutions of Equation (16) are spherical Bessel functions

$$
j_{\ell}(\rho)=\left(\frac{\pi}{2 \rho}\right)^{\frac{1}{2}} J_{\ell+\frac{1}{2}}(\rho)
$$

and the spherical Neumann functions 


$$
\eta_{\ell}(\rho)=(-1)^{\ell+1}\left(\frac{\pi}{2 \rho}\right)^{\frac{1}{2}} J_{-\ell-\frac{1}{2}}(\rho)
$$

Just as in the case of the free particle the condition that $R_{l}(\rho)$ must be finite everywhere including the base of the quantum well (origin or centre of the electron)restricts us to only spherical Bessel functions, $j_{l}$ (Equation (17)) and we have inside the well

$$
R_{E, \ell}(r)=A j_{\ell}(K r) ; r<r_{e}
$$

where $A$ is complex constant and can be determined by normalizing the eigen functions $R_{E, \ell}(r)$. With our conjecture of fractional quantization on the surface of an electron due to over whelming swirling effect and indeed to shallowing of quantum well, we can determine constant " $A$ " of Equation (19) by considering

$$
\phi_{n_{f}}^{\ell}(r)=r_{e}^{\ell} \times \mathrm{e}^{-\left(\frac{h q}{\sqrt{2}}\right)^{2}}, r=r_{e}, \quad \text { [Equation (13)] }
$$

by using momentum operator for fractional charge quantization

$$
\begin{gathered}
\left\langle\phi_{n_{f}}^{\ell}(r)\right\rangle=\frac{\int_{-\infty}^{\infty} r_{e}^{* \ell}\left(i \hbar \frac{\partial}{\partial r} r_{e}^{\ell}\right) \mathrm{e}^{-\left(\frac{h q}{\sqrt{2}}\right)^{2}} \mathrm{e}^{\left(\frac{h q}{\sqrt{2}}\right)^{2}} \mathrm{~d} s}{\int_{-\infty}^{\infty} r_{e}^{* \ell} r_{e}^{\ell} \times \mathrm{e}^{-\left(\frac{h q}{\sqrt{2}}\right)^{2}} \times \mathrm{e}\left(\frac{h q}{\sqrt{2}}\right)^{2} \mathrm{~d} s} ; r=r_{e} \\
=i \hbar \ell \cdot r_{e}^{* \ell}=i \hbar \ell r_{e}^{-\ell}=\frac{i \hbar \ell}{r_{e}^{\ell}} \\
A \equiv\left\langle\phi_{n_{f}}^{\ell}\right\rangle=\frac{i \hbar \ell}{r_{e}^{\ell}}
\end{gathered}
$$

Putting Equation (20) in Equation (19), we have

$$
R_{E, \ell}(r)=\frac{i \hbar \ell}{r_{e}^{\ell}}\left(\frac{\pi}{2 K r}\right)^{\frac{1}{2}} J_{\ell+\frac{1}{2}}(K r) ; r \leq r_{e}
$$

Equation (21) represents the eigenfunction of the swirling strips of the whirl pool in the quantum well. Using Equation (15) in Equation (17) and modifying Equation (21), we have

$$
R_{E, \ell}(r)=\frac{i \hbar \ell}{r_{e}^{\ell}} j_{\ell}(K r) ; r<r_{e}
$$

For $\ell \neq 0,|i| \rightarrow 1$, Equation (22) becomes case II $\left(r<r_{e}\right)$.

\subsection{Case II $\left(r<r_{e}\right)$}

$$
R_{E, \ell}(r)=\hbar \ell r_{e}^{-\ell}\left(\frac{\pi}{2 K r}\right)^{\frac{1}{2}} J_{\ell+\frac{1}{2}}(K r) ; r<r_{e}
$$

The value of $K$ expressed in Equation (3) will further be modified for Equation (23)

$$
K=\left[\frac{2 \mu}{\hbar^{2}}\left(E+V_{0}\right)\right]^{\frac{1}{2}} \text { where } V_{0}=-\frac{e^{2}}{r_{e}} \text { and } E=\frac{p^{2}}{2 \mu}=\frac{(\hbar K)^{2}}{2 \mu}
$$

For fractional charge quantization,

$$
E_{n_{f}}=\frac{\left(\hbar k_{n_{f}}\right)^{2}}{2 \mu}=\frac{\hbar^{2} k_{n_{f}}^{2}}{2 \mu},
$$


we have

$$
\begin{aligned}
K & =\left[\frac{2 \mu}{\hbar^{2}}\left(\frac{\hbar^{2} k_{n_{f}}^{2}}{2 \mu}-\frac{e^{2}}{r_{e}}\right)\right]^{\frac{1}{2}} \\
& =\left[k_{n_{f}}^{2}-\frac{2 \mu e^{2}}{\hbar^{2} r_{e}}\right]^{\frac{1}{2}} ; f e^{2} \equiv n_{f} h q \\
& =\left[k^{2_{n_{f}}}-\frac{2 \mu}{\hbar^{2}} \times \frac{n_{f} h q}{r_{e}}\right]^{\frac{1}{2}} ; \hbar=\frac{h}{2 \pi}, 0.1 \leq n_{f} \leq 0.9 \\
& =\left[k_{n_{f}}^{2}-\frac{2 \mu \times n_{f} 2 \pi \hbar q}{\hbar^{2} r_{e}}\right]^{\frac{1}{2}}=\left[k_{n_{f}}^{2}-\frac{4 \pi \mu n_{f} q}{\hbar r_{e}}\right]
\end{aligned}
$$

We consider $e^{2} \equiv n_{f} h q$ for fractional charge quantization and indeed for electrodynamic behaviour with integrated oscillatory characteristic. The energy levels are obtained by requiring that the eigen function $R_{E, \ell}(r)$ [Equation (23)] with Equation (24) and its derivatives be continuous at the discontinuity of the potential well. Thus the logarithmic derivative $\left(\frac{1}{R_{E, \ell}}\right)\left(\frac{\mathrm{d} R_{E, \ell}}{\mathrm{d} r}\right)$ must be continuous at $r=r_{e}$. For $r>r_{e}$, Equation (1) with $V_{0}=0$ is identical to a free particle, but $E<0$. It is convenient to write

$$
\begin{aligned}
E=-\left(\frac{\hbar^{2}}{2 \mu}\right) \lambda ; \quad E=E_{\text {op }}, \lambda \equiv \nabla \text { so that } & \\
& \lambda=\left(\frac{-2 \mu E}{\hbar^{2}}\right)^{\frac{1}{2} .}
\end{aligned}
$$

Equation (1) with $V_{o}=0$ can be put in the form of spherical Bessel equation by changing $\rho=i \lambda r$ which amounts to replacing $K$ in Equation (15) by $i \lambda$. The domain of $\rho$ does not extend down to zero, as a consequence of which, we take a linear combination of the functions $j_{\ell}$ (Equation (17)) and $n_{\ell}$ (Equation (18)). Using the asymptotic formulas for spherical Bessel and Neumann functions with $\rho \rightarrow \infty$

$$
\begin{gathered}
j_{\ell}(\rho) \rightarrow \frac{1}{\rho} \sin \left(\rho-\frac{\ell \pi}{2}\right) \\
\eta_{\ell}(\rho) \rightarrow-\frac{1}{\rho} \cos \left(\rho-\frac{\ell \pi}{2}\right)
\end{gathered}
$$

The asymptotic behaviour of the spherical Hankel functions of first and second kind with $\rho \rightarrow \infty$ are

$$
\begin{gathered}
h_{\ell}^{(1)}(\rho) \rightarrow-i \frac{\exp \left[i\left(\rho-\frac{\ell \pi}{2}\right)\right]}{\rho} \\
h_{\ell}^{(2)}(\rho) \rightarrow i \frac{\exp \left[i\left(\rho-\frac{\ell \pi}{2}\right)\right]}{\rho}
\end{gathered}
$$

With $\rho=i \lambda r$, we observe that for large $r$, i.e., $r \gg r_{e}$, the functions $j_{\ell}(i \lambda r), \eta_{\ell}(i \lambda r)$ and $h_{\ell}^{(2)}(i \lambda r)$ increases exponentially like $\frac{\exp (\lambda r)}{r}$ and must, therefore, be excluded. The admissible solution is $h_{\ell}^{(1)}(i \lambda r)$, which is proportional to $\frac{\exp (-\lambda r)}{r}$ for $r \gg r_{e}$ because $\exp (-\lambda r)$ is a convergent series. Hence, the desired 
solution of Equation (1) with $V_{o}=0$, i.e., out side the quantum well, is case III $\left(r \gg r_{e}\right)$.

\subsection{Case III $\left(r \gg r_{e}\right)$}

$$
R_{E, \ell}(r)=B h_{\ell}^{(1)}(i \lambda r)=B\left[j_{l}(i \lambda r)+\eta_{l}(i \lambda r)\right] ; r \gg r_{e}
$$

where $B$ is a complex constant and can be determined by normalizing the eigenfunction $R_{E, \ell}(r)$. At $r \gg r_{e}$, we found the normalized eigen function [6], i.e.,

$$
\psi(r, \phi)=\sum_{r=0}^{\infty} r^{2 n_{f}} \sqrt{\frac{\left(4 n_{f}+1\right)}{r_{e}^{4 n_{f}+1}}} \mathrm{e}^{ \pm i m \phi}
$$

with theoretical eigenvalues

$$
E_{n_{f}}=\frac{\left(n_{f}+2\right)\left(n_{f}+1\right) \hbar^{2}}{2 \mu \sin ^{4} \theta} ; 0.1 \leq n_{f} \leq 0.9
$$

The complex constant $B$ in Equation (28) at $r \gg r_{e}$ is equivalent to

$$
B=\frac{2 n_{f} !}{(-1)^{n_{f}+2} \beta^{n_{f}}} \sqrt{\frac{\left(4 n_{f}+1\right)}{r_{e}^{4 n_{f}+1}}}
$$

with

$$
\beta=\frac{\beta_{1}}{\sin ^{2} \theta}, \beta_{1}=\frac{2 \mu E \sin ^{2} \theta}{\hbar^{2}}, \beta=\frac{2 \mu E}{\hbar^{2}} \Rightarrow \beta^{\frac{1}{2}}=\frac{\sqrt{2 \mu E}}{\hbar} \equiv k
$$

(for a free particle).

Remember $\theta \equiv \alpha$, in our present case, and can be used to locate the effect of sub quanta and fractional charge quantization at $r \gg r_{e}$. Equation (29) can be used to monitor the effect of fractional charge quantization in terms of azimuthal angle $\phi$ and also in terms $\theta \equiv \alpha$ for each radial strip of the electron. By applying the conditions of continuity to Equation (23) and Equation (28), we have

$$
\left[\frac{\mathrm{d} j_{\ell}(K r) / \mathrm{d} r}{j_{\ell}(K r)}\right]_{r=r_{o}}=\left[\frac{\mathrm{d} h_{\ell}^{(1)}(i \lambda r) / \mathrm{d} r}{h_{\ell}^{(1)}(i \lambda r)}\right]_{r=r_{o}}
$$

Equation (32) is the transcendental equation for arbitrary $\ell$. In an ideal case, for $\ell=0$, we shall have no surface fractional charge quantization, as a result of which, the attractive electrodynamic potential well dominate over the centrifugal potential and the quantum well becomes more deep with radius of the electron becoming more smaller (charge, i.e., density will increase within a smaller volume). Hence, with $\ell=0$, we can determine the strength of the potential well, i.e., quantum potential well with in an electron, are

$$
j_{o}(\rho)=\frac{\sin \rho}{\rho}, \eta_{o}(\rho)=-\frac{\cos \rho}{\rho}, h_{o}^{(1)}(i \lambda r)=-\frac{1}{\lambda r} \mathrm{e}^{-\lambda r}
$$

and

$$
K \cot K r_{e}=-\lambda
$$

Equation (34) refers to odd parity solution to quantum potential well. Thus, setting $\xi=K r_{e}$ and $\eta=\lambda r_{e}$, and using Equations (24) and Equation (25)

$$
\xi \cot \xi=-\eta
$$

with

$$
\xi^{2}+\eta^{2}=\frac{2 \mu}{\hbar^{2}} V_{o} r_{e}^{2}=\gamma^{2}
$$

where $\left(\frac{2 \mu V_{o} r_{e}^{2}}{\hbar^{2}}\right)^{\frac{1}{2}}$ is the strength parameter of quantum well of an electron. There is no $(\ell=0)$ bound state 
if $\gamma<\frac{\pi}{2}$ (boundary condition of Fractional Fourier Transform (FRFT)) but one $(\ell=0)$ bounds state exists if $\frac{\pi}{2}<\gamma \leq \frac{3 \pi}{2}$, and so on

$$
V_{o}=-\frac{e^{2}}{r_{e}}=\frac{-n_{f} h q}{r_{e}}=\frac{-n_{f} 2 \pi \hbar q}{r_{e}}
$$

(by ignoring the negative sign because it shows attractive electrodynamic potential) in

$$
\begin{gathered}
\gamma=\left(\frac{2 \mu V_{o} r_{e}^{2}}{\hbar^{2}}\right)^{\frac{1}{2}}=\left(\frac{2 \mu r_{e}^{2} \times n_{f} 2 \pi \hbar q}{r_{e} \hbar^{2}}\right)^{\frac{1}{2}}=\left(\frac{4 \pi \mu r_{e} n_{f} \hbar q}{\hbar^{2}}\right)^{\frac{1}{2}} \\
\Rightarrow \gamma_{n f}=\frac{2}{\hbar}\left(\pi \mu r_{e} n_{f} \hbar\right)^{\frac{1}{2}}, 0.1 \leq n_{f} \leq 0.9
\end{gathered}
$$

Equation (38) shows the strength of the quantum well at different depths for whirling strips corresponding to fractional quantized states inside the electron.

\section{Conclusion}

The energy profile (eigenfunctions) of the quasi particle nature of electron quanta is obtained. These energy profiles of stretched electron quanta with sub-quanta (twiggs) on the lateral surface in the momentum space due to twisting (gyroscopic behaviour) will pave a new dimension in theoretical physics to let us know about how particles and sub particles settle in the lowest energy state. With our conjuncture of fractional charge quantization, the shape of the energy profile and energy eigenvalues above the surface of quasi particle nature of an electron quanta in chromotized water sample (confirmed with absorption and Fourier transform inferred (FTIR) spectroscopies) is verified. The other results of energy profiles such as on the surface and inside the surface of quasi particle behaviour of an electron quanta will decipher new physics for understanding the complex nature of exchange energy fields, may be the intermediatory nature of strong forces in the nucleus of the atom due to quarks.

\section{References}

[1] Griffths, D.J. (2005) Introduction to Quantum Mechanics. 2/e, Pearson Education.

[2] Liboff, R.L. (2003) Introduction to Quantum Mechanics. 4/e, Pearson Education.

[3] Gasiorowicz, S. (2003) Quantum Mechanics. 3/e, John Wiley, New York.

[4] Gormani, M., Rehman, F., Ahmed, M.A. and Raza, S.M. (2006) Quantum Behaviour of Dielectric in Dolomite of Balochistan, Pakistan. Journal of the Chemical Society of Pakistan, 28, 414-416.

[5] Yousaf, S., Raza, S.M., Masoom, Y. and Mujeeb, U.R. (2008) Absorption of Radiant Energy in Water: A New Conjecture and Theory of Charge Quantization in Chromotized Water Samples. Science International Lahore. Pakistan, 20, 189-195.

[6] Yousaf, S., Raza, S.M. and Ahmed, M.A. (2008) Newly Developed Recursive Relationship for Fractional Quantum States and Associated Energy Eigen Values. Science International Lahore. Pakistan, 20, 255-260.

[7] Yousaf, S., Raza, S.M., Masoom, Y. and Mujeeb, U.R. (2009) Quantum Theory of Dielectric and Its Applications to Dolomite of Balochistan, Pakistan. Science International Lahore. Pakistan, 21, 29-32.

[8] Iqbal, S., Sarwar, F., Raza, S.M. and Rehman, A. (2015) How Fractional Charge on an Electron in the Momentum Space Is Quantized? ASRJETS, 14, 265-272.

[9] Iqbal, S. (2012) PhD Thesis Analysis and Applications of Fractional Fourier Transform. Unpublished, University of Balochistan, Quetta. 